Brit. J. industr. Med., 1951, 8, 1.

\title{
SPEED AND LOAD STRESS IN A SENSORI-MOTOR SKILL*
}

\author{
BY \\ R. CONRAD \\ From the Psychological Laboratory, University of Cambridge
}

(RECEIVED FOR PUBLICATION AUGUST 20, 1950)

Experimental psychology has long been dominated by the proposition that a knowledge of sensory thresholds would provide a framework for the study of human behaviour. Some serious shortcomings of the traditional psychophysical methods for ascertaining these thresholds have been pointed out by Bartlett (1947), who stressed the fact that the discrete stimulus-response situation of psychophysical experiments can provide little information - about the sensory processes involved in life, where the subject is dealing always with a series of stimuli.

At the same time, psychology has been equally guilty of failing to recognize that under natural conditions perception of a single series of stimuli is not much more than a theoretical possibility. In fact, behaviour must be determined by the perception not only of concurrent series of stimuli in different sensory modes, but also of concurrent, but not necessarily related, series of stimuli in the same mode. Particularly one cannot assume that any one sensory threshold determines the framework of behaviour without taking into account the operation of the other thresholds which are almost certain to be present.

It is almost a century ago that the philosopher William Hamilton (1859) attempted to analyse the numerical limit beyond which attention could not be stretched and proposed an experimental technique which led to an immense number of researches into the span of apprehension.

This field of research has yielded a great deal of information relating to the spatial arrangements, with particular reference to groupings, of static visual displays. But the data which it can provide must assume that the parts of the display being apprehended remain in a constant attentional relationship. However true this may be in some situations, it is obvious that even in the most mundane forms of behaviour, such as cycling in

\footnotetext{
*The work reported in this paper was undertaken for the Human Factors Panel of the Government Committee on Industrial Productivity.
}

B traffic, the attentional demands of the different parts of the perceptual field are continually changing relative to one another.

It is not therefore surprising that side by side with experiments on the span of apprehension there proceeded others which attempted to study the nature of the "division" of attention which appears to occur when an individual adapts to several concurrent but independent series of events. Unfortunately, for every hundred experiments of the former kind there was perhaps one of the latter.

Research in this field began with the experiments of Paulhan (1887) who set himself the task of reciting one poem orally whilst at the same time writing another. From then onwards, the emphasis lay either in the analysis of the effects on performance of a concurrent second task, or on studies of individual differences of ability to perform two tasks at once. Had the same question which inspired the experiments on the span of apprehension been asked-i.e. how many tasks can be simultaneously performed-the early workers might have quickly discovered the presence in their experimental situations of variables which, unrecognized, may have contributed to the relative sterility of research in this important field.

The earliest work of Paulhan (1887), Binet (1890), Jastrow (1891), Sharp (1899), Meumann (1907) and Lorenz (1912), is ably reviewed by McQueen (1917). McQueen himself improved on the techniques of his predecessors and extended their work, but in fact added little new to the meagre body .of knowledge. Nor did later workers, such as Sterzinger (1928), feel the need for any approach substantially different from that introduced nearly half a century earlier.

Concerned as this work has been with the effects of interaction, subjects were never asked to deal with more than two series of events, though there is no obvious theoretical justification for this limit. Nor was it considered necessary to equate the difficulty of the two tasks, except in a single experi- 
ment of McQueen's. Thus no attempt to relate the different measures of interaction obtained could possibly be made.

The typical experiment itself consisted of a bizarre juxtaposition of tasks so that complex mental acrobatics were demanded of the subjects (e.g., card-sorting and counting by $3 \mathrm{~s}$ ) rather than the common and fundamental forms of behaviour necessary for adapting to the changing demands of a physical environment.

In any form of skilled behaviour, if there is a time factor present in the environmental conditions in which it is performed, and the perceptual field is made up of more than one stream of signals, the maintenance of the skill depends in every case on a single dominant perceptual function. This is the ability to determine, before the inherent time limit, which is the next response to be made. It is a commonplace to point out that skill is characterized by a smoothness of performance brought about by selective perception perfectly integrated with unhurried response. But little is understood as yet of the nature of the psychological functions that underlie this ability to perceive selectively, to ignore some signals, to store others, to combine yet others, and to withhold response until the critical moment ; nor do we know a great deal about the factors that disturb the operation of these functions.

What faced both the experimenters on the span of apprehension, and those on the "division" of attention, was the problem of understanding the nature of perceptual complexity, and in a unitary task, the limits beyond which deterioration of performance is inevitable. Hamilton himself had already discovered that far more discrete objects could be apprehended if they were arranged in groups. The two problems therefore cannot be treated in isolation. The workers who followed Paulhan completely failed to realize this, and attempted no control over the complexity of their tasks.

In formulating the problem as a study of interference, an unjustifiable assumption was made of the nature of perceptual complexity in skilled behaviour. Skill was considered to consist of the concurrent performance of tasks which were functionally unrelated and the integration of which was impossible, and indeed absurd.

Clearly until we have some hypothesis as to the nature of perceptual complexity, we can reither control it experimentally. nor attempt to study the limits which can be efficiently organized in the performance of skills. We need to find independent variables that are always part of the perceptual field; we need to discover the variables that directly determine the ease with which the field can be organized so that effective adaptive behaviour can be maintained.

A large number of such variables have been the subject of experiment for very many years. Many factors have been extensively studied, such as the physical intensity of signals, the spatial and (more recently) the temporal relationship both within the field, and between field and observer, also figureground and movement direction relationships, as well as many others.

Much less attention has been paid to the variable that describes the rate at which display changes demanding response occur. The present writer (1949a) and Mackworth (1949) both showed, however, that such speed effects are very marked and constitute a factor in perception of considerable importance. Experiment showed that errors increased steeply out of all proportion to the absolute increase in speed.

The speed variable defines how many signals occur per unit time. Bur it does not describe how they occur. A single source cannot provide two signals simultaneously. But two sources can. If two sources each simultaneously present ten signals * per minute, signals occur at the rate of 20 per minute, and 20 responses per minute may be required. But if two sources each present ten signals per minute, neither simultaneously nor at regular intervals, the perceptual complexity of this latter task is likely to be greater than that of the former, although the average rate of response is the same. To characterize the difference between these two tasks a variable needs to be defined which is descriptive only of the display as a whole, and refers to the number of separate streams of signals that occur independently of each other, but require simultaneous consideration. Mackworth (op. cit.) has called this the "load" factor, and I have further described it (1949b). The load factor is never present in a static display, and there can only be a load variable when time is one of the dimensions of the perceptual situation. Speed and load qualify each other, and a display described in terms of only one of these factors is meaningless.

Evidence that speed and load are psychologically independent was provided by Mackworth (1948), who analysed the effects of these two variables on error scores in a complex intellectual skill. He found a logarithmic relationship between prescribed rate of response and number of errors, but the effects of load on number of errors was in this case best expressed linearly. From this one must conclude that speed and load describe two probably distinct and different features of the physical environment.

The task set by Mackworth was one in which 
the display changes occurred as a result of the subject's response. Responses had to be chosen in such a way that if correct, display changes would develop in a clearly defined way. Incorrect response would lead to errors. The critical feature of the task was that the subject could not think at any convenient rate, but had to respond within time limits controlled by the experimenter. Failure to respond within the limit set resulted in a second source of error. This situation is characteristic of a number of industrial skills in which an operator is required to make a correct decision at the correct moment, little change occurring in the objective situation between responses.

Clearly there are many other skills in which the display changes are themselves largely beyond the control of the operator. In such situations the operator's task is one of constant vigilance and adaptive response to endless changes which occur regardless of his behaviour. In the present experiment the effects of speed and load on performance in the latter kind of task are investigated. The speed range has been extended over that used in my previous experiments, and refined criteria of performance have heen developed.

\section{Apparatus}

The apparatus was that described in previous papers (1949a, 1949b), and a photograph is reproduced in Fig. 1. The original equipment was constructed by Mr. C. B. Gibbs, but to obtain a greater variability in the speed range, the main driving shaft was later powered by a velodyne motor and control unit (not shown in Fig. 1), designed and built by Dr. A. Carpenter. This readily provided any desired speed which, irrespective of mains voltage fluctuations, was maintained with a variation of not more than $\pm 1 \%$.

Each pointer revolved at a constant but different speed from the others. This was determined by the speed of the main drive and by the ratio of the pointer's gear link. Relative pointer speeds are best illustrated as follows. If the speed of the main drive were 1 r.p.m., then the speed of the pointers from left to right would be :

$$
1.25 \text { r.p.m. } \quad 1.15 \text { r.p.m. } 0.90 \text { r.p.m. } 0.75 \text { r.p.m. }
$$

Dial faces and pointers were easily detachable, so that the number of streams of signals could be anything from one to four. The subject's task was to turn the appropriate knob every time any pointer and target mark coincided. A paper record was obtained of missed responses, and also of accuracy of response if this was made at all. As will be seen from the relative pointer speeds, and from the fact that the target marks were equidistantly spaced around the dial faces, it was not possible to learn by heart the required order of response, but instead throughout the test the subject had to estimate which knob was next to be turned, in order to respond at the correct moment. All the perceptual information necessary for this task was fully visible.

The subjects were 20 Naval ratings aged 19 to 23 years.

\section{Procedure}

Subjects were tested with five speeds $(40,60,80$, 100,120 signals per minute), and three loads $(2,3$,

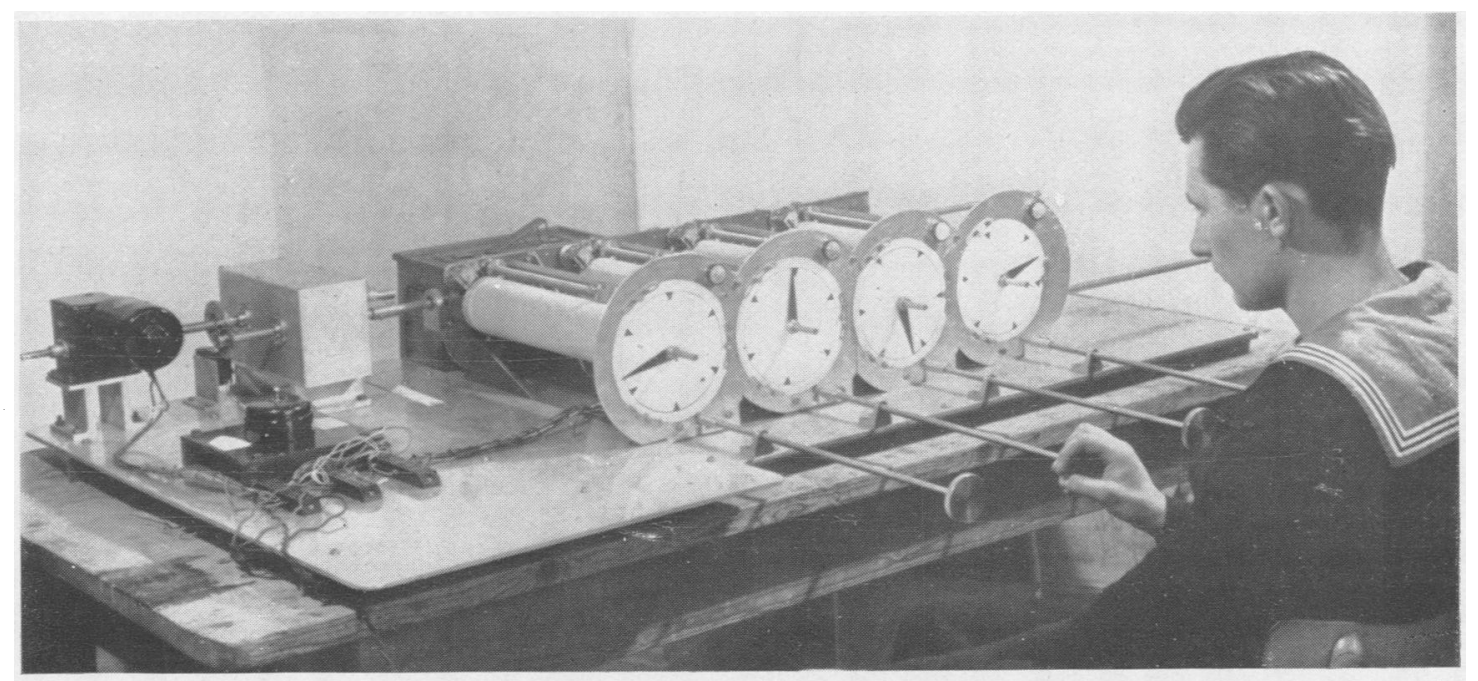

FIG. 1.-Photograph of the apparatus. 
4 dials). In those tests where three dials were used, the extreme left-hand one was removed, the two left-hand ones being removed where a load of two dials was required. There were thus 15 conditions in all. Each subject did a ten-minute test under each condition, doing three tests daily on five consecutive days, the test order being made suitably at random.

\section{Results}

Errors of Omission.-Errors of omission were considered to have occurred when the subject failed completely to respond to a signal. The aptness of this criterion of performance is easily recognized when one remembers how frequently, for example in games, in explaining mistakes one is forced to admit to a simple failure to notice something occur. Failure to respond to a critical signal must be regarded as a fundamental source of error in the breakdown of skill under stress. It is therefore important to know the effects on this error of speed and load increases. These are presented in Table 1 and Fig. 2.

TABLE 1

NUMBER OF SIGNALS MISSED PER MINUTE

\begin{tabular}{|c|c|c|c|c|c|}
\hline & \multicolumn{5}{|c|}{ Signals per Minute } \\
\hline & 120 & 100 & 80 & 60 & 40 \\
\hline $\begin{array}{c}4 \\
\text { Dials }\end{array}$ & $\begin{array}{c}44 \cdot 4 \\
(51 \cdot 9)^{*}\end{array}$ & $\begin{array}{c}25 \cdot 4 \\
(29 \cdot 2)\end{array}$ & $\begin{array}{c}15 \cdot 1 \\
(14 \cdot 5)\end{array}$ & $\begin{array}{c}4 \cdot 9 \\
(5 \cdot 9)\end{array}$ & $\begin{array}{l}1 \cdot 4 \\
(1 \cdot 6)\end{array}$ \\
\hline$\stackrel{3}{\text { Dials }}$ & $\begin{array}{c}31 \cdot 5 \\
(27 \cdot 4)\end{array}$ & $\begin{array}{c}15.9 \\
(13 \cdot 7)\end{array}$ & $\begin{array}{c}8 \cdot 2 \\
(5 \cdot 8)\end{array}$ & $\begin{array}{c}1 \cdot 8 \\
(1.9)\end{array}$ & $\begin{array}{c}0.4 \\
(0.4)\end{array}$ \\
\hline$\stackrel{2}{\text { Dials }}$ & $\begin{array}{c}12 \cdot 8 \\
(11 \cdot 2)\end{array}$ & $\begin{array}{c}4 \cdot 5 \\
(4 \cdot 7)\end{array}$ & $\begin{array}{c}1 \cdot 8 \\
(1 \cdot 6)\end{array}$ & $\begin{array}{c}0.5 \\
(0.4)\end{array}$ & $\begin{array}{c}0 \\
(0 \cdot 1)\end{array}$ \\
\hline
\end{tabular}

* Figures in brackets represent values calculated from the equation.

The striking fact that emerges is that in increasing the speed three times, errors increase at least 30 times with the four-dials display. It is obvious, even with the lightest load, that errors are not directly proportional to speed. The serious effect of speed is felt at the first increase from 40 to 60 signals per minute. With all loads this extra speed gives a statistically significant increase not only in the absolute number of errors, but also in the proportion of presented signals which are missed.

Mackworth (1948) found that in his results the relation between speed and error was expressed by an equation of the form :

$$
\text { (where } \mathrm{N}=\log \mathrm{N}=\mathrm{a}+\mathrm{bS}
$$

This equation, however, is not satisfactory for the data in Table 1, which can best be expressed in the form :

$$
\log \mathrm{N}=\mathrm{a}+\mathrm{b} \log . \mathrm{S} .
$$

This latter is suitable with all three loads, in each case the fit being significant at the 0.001 level of probability. The actual equations are as follows :

$$
\begin{aligned}
& 4 \text { Dials: } \log N=3.2148 \text { Log. } S-5.0075 \\
& 3 \text { Dials: } \log N=4.1037 \text { Log. } S-6.9900 \\
& 2 \text { Dials: } \log N=4.4145 \log . S-8 \cdot 1233 \\
& \text { ( } N=\text { No. of signals missed per minute) } \\
& \text { ( } \mathbf{S}=\text { No. of signals presented per minute) }
\end{aligned}
$$

It may be noted in passing that Fraser (1950) in an aiming task also found a similar relationship

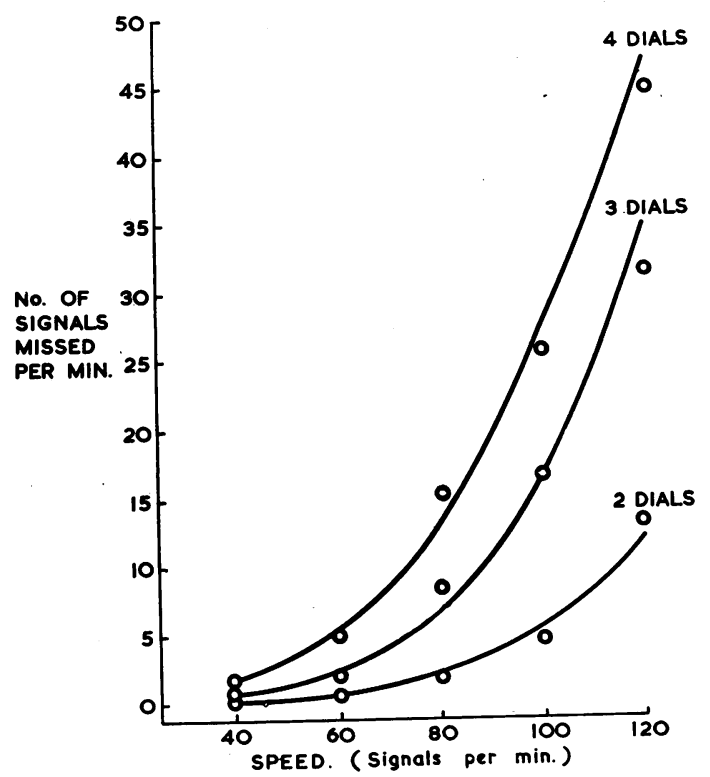

Fig. 2.-Effects of speed stress and load stress on errors of omission.

between speed and accuracy. The utmost care must, however, be used in comparing speed/accuracy relationships in different tasks unless one is quite certain that the type of error referred to is the same in each case. In the two experiments quoted and the present one error means something different in each case. It will be shown later that in this experiment another error criterion behaves quite differently with an increase in speed.

Taking now the effects of load increases with a constant speed, a serious increase is again found in the number of signals missed. Without altering the number of signals presented per minute, doubling the load very much more than doubles the number of errors of omission. Significant 
TABLE 2

EFFECT ON ERRORS OF OMISSION OF SPEED INCREASES AND LOAD INCREASES

\begin{tabular}{|c|c|c|c|c|c|}
\hline Source & $\begin{array}{l}\text { Sum of } \\
\text { Squares }\end{array}$ & df. & $\begin{array}{l}\text { Mean } \\
\text { Square }\end{array}$ & $F$ & $1 \%$ \\
\hline Sp zed $(\mathbf{S})^{*}$ & $3,324,527 \cdot 4$ & 4 & $831,131 \cdot 9$ & $98 \cdot 50$ & $3 \cdot 32$ \\
\hline Load (L)* & $1,022,303 \cdot 6$ & 2 & $511,151 \cdot 8$ & $64 \cdot 32$ & $4 \cdot 60$ \\
\hline Subjects (O) & $403,548 \cdot 8$ & 19 & $21,239 \cdot 4$ & $3 \cdot 97$ & $1 \cdot 99$ \\
\hline $\mathbf{S} \times \mathbf{L} \quad \ldots$ & $624,225 \cdot 4$ & 8 & $78,028 \cdot 2$ & $14 \cdot 60$ & $2 \cdot 51$ \\
\hline $\mathbf{S} \times \mathbf{O} \quad \ldots$ & $641,306 \cdot 5$ & 76 & $8,438 \cdot 2$ & $1 \cdot 58$ & $1 \cdot 35$ \\
\hline $\mathrm{L} \times \mathrm{O}$ & $302,001 \cdot 6$ & 38 & $7,947 \cdot 4$ & 1.49 & $1 \cdot 45$ \\
\hline $\bar{S} \times \mathbf{L} \times \bar{O}$ & $812,408 \cdot 7$ & 152 & $5,344 \cdot 8$ & - & - \\
\hline Total & $7,130,322 \cdot 0$ & 299 & - & - & - \\
\hline
\end{tabular}

${ }^{*}$ The effects $\mathrm{S}$ and $\mathrm{L}$ have been tested against $\mathrm{S} \times \mathrm{O}$ and $\mathrm{L} \times \mathrm{O}$ respectively.

differences occur at the first increase, and it is evident that load alone is an important factor in skill deterioration.

Analysing the variances involved (Table 2) indicates that there is a speed/load interaction which is statistically significant at the $1 \%$ level. Inspection of Table 1 suggests that this interaction is such that the greater the load the more serious will be the speed increases.

A possible regression relation between log errors, $\log$ speed, and $\log$ load was indicated by the fact that both the regression of $\log$ errors on log speed (load constant), and that of log errors on log load (speed constant), were linear. The following equation of a ruled surface provided a satisfactory fit to the observed data.

$(2 \cdot 975-\log N)=5.514(2 \cdot 480-\log S)(1 \cdot 172-\log L)^{*}$ where $\mathbf{N}=$ No. of signals missed per min.

$\mathbf{S}=$ No. of signals presented per min. (speed)

$\mathbf{L}=$ No. of dials (load)

Subtracting the errors of omission from the number of signals presented gives the number of attempts at the target, the shots. These are given in Table 3. The faster speeds acted as an incentive

TABLE 3

NUMBER OF SHOTS ATTEMPTED

\begin{tabular}{l|c|c|c|c|c}
\hline & \multicolumn{4}{|c|}{ Signals per Minute } \\
& 120 & 100 & 80 & 60 & 40 \\
\hline 4 Dials & $75 \cdot 6$ & $74 \cdot 6$ & $64 \cdot 9$ & $55 \cdot 1$ & 38.6 \\
\hline 3 Dials & $88 \cdot 5$ & $84 \cdot 1$ & $71 \cdot 8$ & $58 \cdot 2$ & $39 \cdot 6$ \\
\hline 2 Dials & $107 \cdot 2$ & $95 \cdot 5$ & $78 \cdot 2$ & $59 \cdot 5$ & $40 \cdot 0$ \\
\hline
\end{tabular}

*I am indebted to Mr. W. Donaldson of the Statistical Laboratory, University of Cambridge, for the calculation of this equation. to make more attempts, but it is noticeable that at faster speeds, the increase in shots slows down sharply. Thus with four dials, increasing the speed from 100 to 120 signals per minute, results in an increase of only one extra shot.

It may be noted that during the ten-minute period of any test no consistent deterioration of performance was observed, either in the individual or group average minute by minute records.

Errors of Timing.-Primary interest in further analysis of error lay in observing the effects of speed and load increases on the ability to make response at the correct moment. Bartlett (1943) has pointed out that timing deteriorates with fatigue. But so far there have been no investigations published of the effects of speed stress and load stress on timing.

In this experiment, the correct moment for response was when the moving pointer was perfectly aligned with a target mark. Since the target mark theoretically was a point, every single response would necessarily involve some degree of error. This is made clear to the subjects, and it is essential to adopt this procedure.

If one accepts the alternative method and allows the target to have a certain width which is represented on the display, the target will be of a constant size spatially, but its temporal size will depend on the speed of the pointer. Thus varying the speed will not only alter the available time between signals, but will also affect the size of the target. In such a situation it would not be possible to determine which part either of these two factors played in producing error. In treating every response as necessarily involving some degree of timing error we can eliminate the possible effects of a changing size of target.

A sample of the recorded performance of each subject under each test condition was taken, and the deviation from the target mark measured in seconds. Since the responses which were made too soon were approximately equal to those made too late, the sign plus or minus has been ignored. The resulting mean size of error under each condition of speed and load is shown in Table 4. The figure in each cell is the mean of from 800 to 1,600 responses.

Table 4 illustrates clearly the point made earlier that the effects of speed are not necessarily the same when different criteria of performance are taken. Within the range used, timing is apparently not influenced by changes in the rate at which signals occur. This is particularly interesting when studied in conjunction with Table 3 . Considering performance with a load of four dials, although the fastest actual rate of response is double that of the 
TABLE 4

ERRORS OF TIMING (MEASURED IN SECONDS)

\begin{tabular}{l|c|c|c|c|c|c|c}
\hline & & \multicolumn{5}{|c|}{ Signals per Minute } & Means \\
& & 120 & 100 & 80 & 60 & 40 & \\
\hline 4 Dials .. & 0.25 & 0.27 & 0.28 & 0.25 & 0.27 & 0.26 \\
\hline 3 Dials .. & 0.19 & 0.21 & 0.20 & 0.18 & 0.20 & 0.20 \\
\hline 2 Dials .. & 0.12 & 0.12 & 0.13 & 0.12 & 0.11 & 0.12 \\
\hline Means.. & 0.19 & 0.20 & 0.20 & 0.18 & 0.19 & \\
\hline
\end{tabular}

slowest, the timing error remains constant. It does not seem to matter how short the interval between responses is : if the response is made at all-and we have seen that it sometimes is not-then it is made within constant limits around the exactly correct moment. This does not mean that timing is independent of the internal nature of the skill. For any given speed, varying the load affects the size of timing error. Doubling the load approximately doubles the size of error. Timing therefore is a criterion of skilled performance which is affected by load but not by speed changes. This is further evidence in support of the view that speed and load are psychologically distinct factors.

So far only the mean size of error has been considered. The distribution of the size (in time) of 100 errors is represented in Fig. 3. Fig. 3 (a) shows the effect of load increases keeping speed

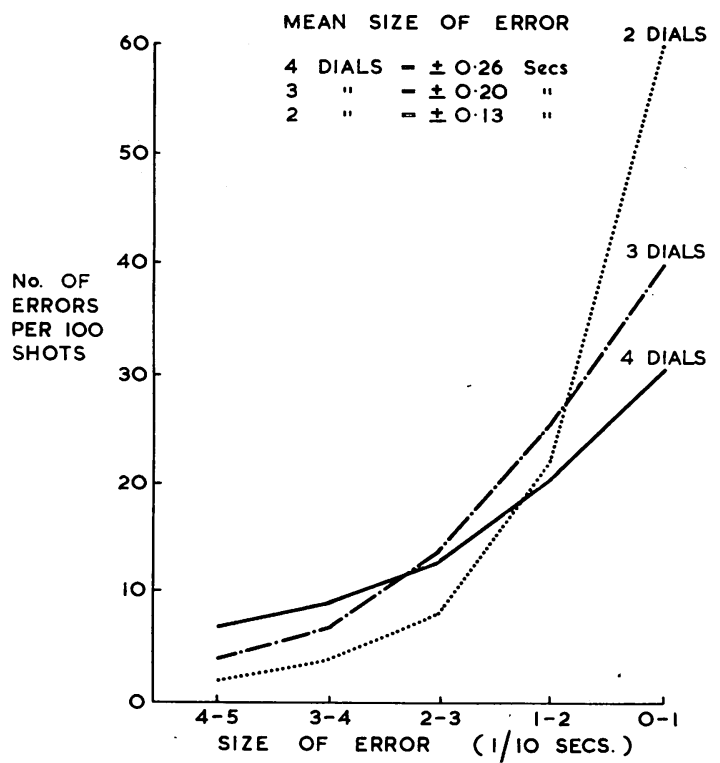

TABLE 5

PERCENTAGE OF SHOTS FALLING INSIDE $\Lambda$ TARGET AREA 0.4 SECONDS WIDE

\begin{tabular}{l|c|c|c|c|c}
\hline & \multicolumn{6}{|c}{ Signals per Minute } \\
\hline & 120 & 100 & 80 & 60 & 40 \\
\hline 4 Dials & $54 \cdot 2$ & $50 \cdot 8$ & $48 \cdot 7$ & $55 \cdot 9$ & $50 \cdot 8$ \\
\hline-1 Dials & $66 \cdot 1$ & $61 \cdot 8$ & $64 \cdot 2$ & $68 \cdot 4$ & $66 \cdot 9$ \\
\hline 2 Dials & $83 \cdot 2$ & $83 \cdot 5$ & $84 \cdot 1$ & $80 \cdot 8$ & $86 \cdot 0$ \\
\hline
\end{tabular}

constant, and Fig. 3 (b) the effect of speed keeping load constant.

The differences in the two sets of distributions are marked. For any given speed, the effect of increasing the load lies mainly in the fact that the subject accepts a lower standard of what is worth trying for with the result that his responses spread out further and further from the target point. Increasing the speed with any one load does not show this effect. The distribution of errors remains fairly constant. This is an interesting confirmation of a point, incidental to her main enquiry, noted by Vince (1948). She found, when the speed of a tracking course was increased, that there was a very high correlation between mean space error in tracking and the rate of the course., i.e., the mean error of timing was constant. The general effect of this is shown in Table 5. In this table in effect

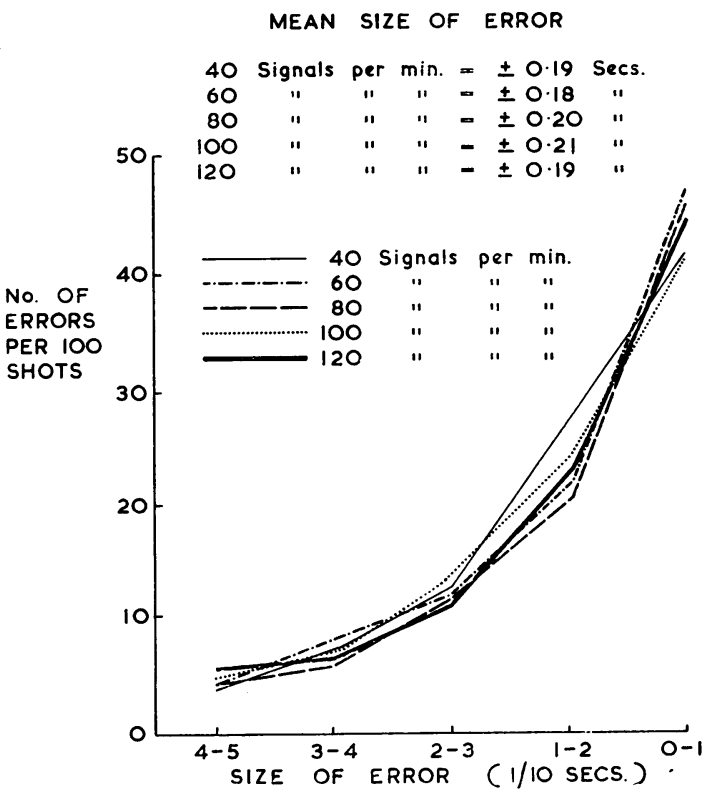

Figs. 3a and 3b.--Effects of speed stress and load stress on errors of timing. 
the practical question is asked. Suppose that the critical period of safety was one-fifth of a second on either side of the target, that a response made within that period was adequate, but that any made outside the period would lead to serious error, under different conditions, what proportion of the responses would be "safe"? The trend is clear. Speed increases alone would make little difference. But increasing the load markedly reduces the proportion of "safe" responses.

\section{Conclusions}

In view of the exploratory nature of this experiment, it is felt that the presentation of the results should not at this stage be accompanied by an inevitably speculative discussion of their significance in the general study of sensori-motor skill. In general these results reinforce the inference drawn from Mackworth's study referred to earlier. Speed and load appear to describe environmental factors present in such skills, which have different causes, and the psychological effects of which are to some extent independent. This inference must be considered of great importance in view of the frequent confusion of these variables.

Any further conclusions must necessarily be stated in terms of problems to be solved. In particular we need to understand the factors which limit the rate at which responses are made, and the factors which influence the individual's ability to respond within fixed time limits. Both of these problems should be amenable to experimental investigation, and indeed have some urgency as the study of the nature of skilled behaviour develops. An experimental analysis of the cause of the changes in performance is therefore now in progress.

\section{Summary}

In this paper previous work on the distribution and division of attention is critically reviewed from the point of view of its relevance to the study of skill. It is felt that fundamental methodological weaknesses minimize its value to this field of research. In particular, the presence of time as an environmental factor in skilled behaviour has been consistently underrated.

Time is effective in skill in the form of speed.
This in the simplest terms can be described as the rate at which critical display changes occur. Speed, however, needs to be qualified by another factor, load, which describes the number of independent streams of signals which comprise the changing display.

An experiment was carried out to study the effects on skilled performance of increases of speed and load beyond the point at which deterioration occurs. Twenty subjects did the same task, which demanded adaptation to a continuously changing situation, under three different conditions of load, and at five different speeds for each load.

The results can be summarized as follows :-

(a) The number of siznals not responded to at all bears a logarithmic relationship to the rate at which they are presented. (b) At any one speed, the incidence of this type of error depends markedly on the load content of the display. (c) Statistical interaction between speed and load effects is demonstrated. (d) Of the responses made, the size of errors of timing is not affected by the speed of the task. But doubling the load approximately doubles the timing error.

I should like to express my thanks to Professor Sir Frederic Bartlett, whose influence throughout this paper is so evident. I also have a debt to Dr. N. H. Mackworth for his continuous advice, and to Miss V. R. Cane and Mr. D. E. Broadbent for many valuable suggestions.

\section{REFERENCES}

Bartlett, F. C. (1943). Proc. roy. Soc. B., 131, 247.

Binet 1947). Brit. med. J., 1, 835.

Binet, A. (1890). Rev. Philos., 29, 138.

Conrad, R. (1949a). "The effects of Very Fast Speeds on Multiple Dial Watching ", Med. Res. Coun. Appl. Psychol. Unit, No. 115. 1949b). "Preliminary Analysis of the Load Factor in Multiple Dial Watching ". Ibid., No. 114, (not generally circulated).

Fraser, D. C. (1950). “The Speed and Accuracy of Plotting with a Simulated P.P.I. Marker Strobe. Part II-Relation of Speed and Accuracy". Mbil., No. 128 50.

Hamilton, W. (1859). "Lectures on Metaphysics", Vol. 1. (Lecturc 14). Edinburgh.

Jastrow, J. (1891). Amer. J. Psychol, 4, 219.

Lorenz, J. (1912). Arch. ges. Psychol., 24, 313.

Mackworth, N. H. (1948). Unpublished thesis. (1949). Nature, London, 164, 982.

McQueen, E. N. (1917). “Brit. J. Psychol. Monog. Suppl., 2, No. 5.

Meumann, E. (1907). "Vorlesungen zur Einführung in die experimentelle Pädagogik und ihre psychologischen Grundlagen ". Leipzig.

Paulhan, F. (1887). Rev. Sci., Paris, Ser., 3, 13 (An. 24), 684.

Sharp, S. E. (1899). Amer. J. Psychol. i0, 329.

Sterzinger, O. (1928). Z. angew. Psychol., 29, 177

Sterzinger, O. (1928). Z.: angew. Psychol., 29, 177. and the Psychological Refractory Period ". Brit. J. Psychol. 38, 3,149 . 